\title{
BMJ Open Values, preferences and burden of treatment for the initiation of GLP-1 receptor agonists and SGLT-2 inhibitors in adult patients with type 2 diabetes: a systematic review
}

\author{
José Gerardo González-González, ${ }^{1,2}$ Alejandro Díaz González-Colmenero, ${ }^{1}$ \\ Juan Manuel Millán-Alanís (D) , ${ }^{1}$ Lyubov Lytvyn, ${ }^{3}$ Ricardo Cesar Solis (D) , \\ Reem A Mustafa, ${ }^{4}$ Suetonia C Palmer, ${ }^{5}$ Sheyu Li, ${ }^{6,7}$ Qiukui Hao (D) , \\ Neri Alejandro Alvarez-Villalobos, ${ }^{1}$ Per Olav Vandvik, ${ }^{9}$ \\ René Rodríguez-Gutiérrez (D) ${ }^{1,2}$
}

To cite: González-González JG, Díaz González-Colmenero A, Millán-Alanís JM, et al. Values, preferences and burden of treatment for the initiation of GLP-1 receptor agonists and SGLT-2 inhibitors in adult patients with type 2 diabetes: a systematic review. BMJ Open 2021;11:e049130. doi:10.1136/ bmjopen-2021-049130

- Prepublication history and additional supplemental material for this paper are available online. To view these files, please visit the journal online (http://dx.doi.org/10.1136/ bmjopen-2021-049130).

Received 20 January 2021 Accepted 19 March 2021

D) Check for updates

(c) Author(s) (or their employer(s)) 2021. Re-use permitted under CC BY-NC. No commercial re-use. See rights and permissions. Published by BMJ.

For numbered affiliations see end of article.

\section{Correspondence to} Dr René Rodríguez-Gutiérrez; rodriguezgutierrez.rene@mayo. edu

\section{ABSTRACT}

Objectives Assess values, preferences and burden of treatment that patients with type 2 diabetes consider when initiating glucagon-like peptide- 1 receptor agonists (GLP-1 RA) or sodium-glucose cotransporter-2 inhibitors (SGLT-2i) compared with other glucose-lowering options. Methods Paired reviewers independently included studies reporting quantitative or qualitative methods to assess values, preferences and burden of treatment reported by patients with type 2 diabetes regarding the initiation of GLP-1 RA or SGLT-2i over other alternatives. A systematic search in MEDLINE, Scopus, EMBASE, Web of Science and Cochrane Central Register of Controlled Trials from inception until May 2020 was performed by an experienced librarian. Risk of bias was assessed with a specifically designed tool for values and preferences studies.

Results 17 studies (7296 patients) proved eligible. Studies fulfilling criteria for SGLT-2i were not identified. Five studies (2662 patients) evaluated preferences for GLP-1 RA compared with other glucose-lowering medications. 12 studies (4634 patients) evaluated preferences between, at least, two kinds of GLP-1 RA or their injection devices based on the following attributes: efficacy, dose, application frequency, device characteristics. Among studies comparing GLP-1 RA to other glucose-lowering medications, some preferences were observed for dypeptil peptidase- 4 inhibitors compared with once daily liraglutide. Comparing different attributes of GLP-1 RA drugs and devices, cardiovascular risk reduction, glucose lowering potential, once weekly and simple administered regimens were the most preferred. Conclusions As no evidence for preferences on SGLT2i was available, only preferences for GLP-1 RA were assessed; however, evidence is still limited for the latter. Studies comparing preferences for GLP1-RA to other glucose-lowering alternatives only included twice daily or once daily injection regimens of GLP-1 RA drugs. According to our findings, once weekly alternatives are widely preferred than the formers. The extent to which
Strengths and limitations of this study

In the design of the search strategy, we employed a previously published filter for studies evaluating values and preferences.

- Risk of bias assessment of included studies was performed in accordance with a specific tool for assessing values and preferences studies.

- The GRADE approach was employed to evaluate the certainty of our results.

- Results are mostly based on studies graded at high risk of bias.

- We did not found studies evaluating preferences for initiation of sodium-glucose cotransporter-2 inhibitors.

patients with type 2 diabetes value reduced adverse cardiovascular and kidney outcomes, weighed benefits against harms and burden of treatment is limited and with very low certainty.

PROSPERO registration number CRD42020159284.

\section{BACKGROUND}

The American Diabetes Association and the European Association for the Study of Diabetes have highlighted the importance of providing a patient-centred approach in patients with type 2 diabetes. ${ }^{1}$ To support clinicians in providing holistic care, it is important to understand the values and preferences that are considered by patients when choosing a particular treatment option. ${ }^{2}$ More specifically, evidence on how patients weigh the balance of benefits, harms and burden of treatment can inform patientcentred practice. 
Box 1 Linked resources in the BMJ rapid recommendations cluster

Reference to this values and preferences systematic review here.

- Reference to guideline paper ${ }^{37}$

- Reference to prognostic systematic review ${ }^{38}$

- Reference to systematic review and network meta-analysis for SGLT-2i and GLP-1 receptor agonists for type 2 diabetes $^{39}$

- Reference to MAGICapp public guideline: to appear at www.magicapp.org

- Reference to MAGIC multiple comparisons evidence summaries and decision aids: www.magicevidence.org/match-it

Glucagon-like peptide-1 receptor agonists (GLP-1 RA) and sodium-glucose cotransporter-2 inhibitors (SGLT2i) are two new drug classes of medications to treat type 2 diabetes that are rapidly changing clinical practice because of demonstrable reductions in cardiovascular and kidney outcomes, without increasing hypoglycaemic. ${ }^{3-10}$ These drugs have notable differences in their benefits and harms and how patients are required to administer them. While GLP-1 RA are mostly injected, SGLT-2i are taken orally. The extent to which these treatments impact patients and carers (treatment burden) is often ignored both in the clinical decision-making process and clinical practice guidelines. Moreover, understanding the values and preferences that patients consider in the process of initiating either of both therapies is still inconclusive, and a thorough and integrative analysis of the available evidence could assist both patients and clinicians in the integral management of the disease. ${ }^{11}$

As a result of the aforementioned, we performed this systematic review to inform a clinical practice guideline (BMJ Rapid Recommendation) on the values and preferences that patients consider in the process of initiating GLP-1 RA and SGLT-2i when compared with each other or other drug treatments for type 2 diabetes (box 1). The goal of the BMJ Rapid Recommendations project is to create rapid and trustworthy recommendations regarding medical topics of interest by identifying relevant studies which might change practice and are of interest to readers. ${ }^{12}$ These guidelines were also informed by a linked systematic review and network meta-analysis on effectiveness and a systematic review on risk prediction models. Together these reviews confirmed, with overall high certainty evidence, benefits of SGLT-2i and GLP-1 RA while demonstrating that absolute benefits differ across patients with different risks for cardiovascular and renal outcomes. In this context, our systematic review was performed to inform judgements on the values that patients consider when balancing benefits, harms and burdens of treatment for SGLT-2i and GLP-1 RA.

\section{METHODS}

We followed the Preferred Reporting Items for Systematic Reviews and Meta-Analyses checklist for writing this review. $^{13}$

\section{Eligibility criteria}

We included any study design using quantitative or qualitative analysis to report values and preferences held by patients with type 2 diabetes mellitus when initiating GLP-1 RA or SGLT-2i treatments or alternative glucoselowering therapy. We excluded: (1) cost-effectiveness studies (as preferences are not directly assessed), (2) studies that report data that is not patient-reported (as they do not reflect the overall patient perspective), (3) studies assessing patient satisfaction on a specific treatment rather than preferences for it when compared with other choices, (4) studies that elicited or explored treatment preferences without reporting the process or factors considered in the decision (as results could be biased due to lack of assessment of values driving the preference), (5) studies of patients with a previously stated preference for GLP-1 RA or SGLT-2i (as results can be biased toward one treatment choice due to previous experience with it) and (6) randomised clinical trials that evaluated patient preferences of a given intervention over a previous treatment (due to possible differences in experiencing each treatment).

\section{Search strategy}

A systematic search strategy was performed on MEDLINE, Scopus, EMBASE, Web of Science and the Cochrane Central Register of Controlled Trials from inception until May 2020. An experienced search specialist designed and conducted the search strategy using a combination of keywords and Medical Subject Headings terms related to values and preferences considered by patients with type 2 diabetes mellitus for initiating GLP-1 RA or SGLT-2i (online supplemental material 1). A previously published filter for studies regarding values and preferences was added to narrow the obtained studies. ${ }^{14}$

\section{Study selection}

After excluding duplicated studies, three reviewers independently and in duplicate screened the title and abstract of retrieved records. Potentially eligible reports were then reviewed in full text. Differences were reconciled by either consensus or discussion with a third reviewer. To ensure an adequate inter-rater agreement, the investigators performed calibration exercises until acceptable agreement was achieved with Cohen's kappa coefficient $>0.7$. Study selection process was performed in the Distiller Systematic Review Software (Evidence Partners DistillerSR, Ottawa, Canada).

\section{Data collection}

A web-based extraction form for data collection was used following piloting to ensure adequate inter-rater agreement and later modifications according to reviewers' input. Paired data extractors worked independently to abstract study characteristics, participants' baseline characteristics, methods used to measure values and preferences, and number and percentage of patients who chose to take the medication according to their values and 
preferences. Disagreements in the data collection process were resolved by either consensus or arbitration by a third reviewer.

\section{Outcome definition}

The term 'values and preferences' was defined according to the Grading of Recommendations, Assessment, Development and Evaluation (GRADE) working group definition: 'the process that individuals use in considering the potential benefits, harms, costs, limitations and inconvenience of the management options in relation to one another' ${ }^{15}$ In order to broaden our scope, the following definition was also considered: 'given a choice, the selection of one alternative a priori'. ${ }^{16}$ We considered reporting of the following attributes: benefits, harms, costs, limitations or inconvenience related to available treatment options.

\section{Risk of bias assessment}

Two independent reviewers working in duplicate adjudicated risks of bias in individual studies based on our main outcome, using a tool proposed by the GRADE working group. It evaluates the following four domains: selection of participants into the study, completeness of data measurement instrument and data analysis. ${ }^{17}$ Disagreements were resolved by consensus or arbitration by a third reviewer.

\section{Certainty of evidence assessment}

To assess the certainty of evidence for the different drug profile comparisons that were included in this review, we followed the constructs proposed by the GRADE working group which are: study design, risk of bias, inconsistency, indirectness, imprecision and other methodological considerations. An overall certainty of evidence grade was then obtained (very low, low, low-moderate, high). ${ }^{18}$

\section{Data synthesis}

Due to the nature of the research question and design of the included studies, our results are reported as a narrative synthesis since a pooled analysis is not feasible.

\section{Patient and public involvement}

Patients or members of the public were not involved with the design of this study.

\section{RESULTS}

\section{Search strategy and study selection}

A total of 11162 records were retrieved in the search and screened using the title and abstract. (figure 1) From these, 86 full-text articles were assessed for eligibility and 17 studies comprising 7296 patients were included in this review $^{19-35}$ (table 1$)$. We did not identify studies reported values and preferences of SGLT-2i and all eligible studies evaluated GLP-1 RA.

\section{Study characteristics}

All studies employed quantitative methods to assess outcomes of interest. Five studies comprising a total of 2662 patients evaluated preferences for GLP-1 RA versus other glucose-lowering drugs. ${ }^{19-23}$ Furthermore, 12 studies comprising a total of 4634 patients evaluated preferences between, at least, two different GLP-1 RA medications or related injection devices, taking into account clinical attributes and/or device-related ones such as dosing, application frequency or characteristics of the application device. ${ }^{24-35}$ Mean age of participants in the included studies ranged between 52.7 and 63.9 years. Most studies reporting duration of diabetes and included patients at least 1 year after diagnosis.

\section{Employed methodologies to elicit values and preferences}

The most frequently employed methodology to elicit patients' preferences was discrete choice experiment (DCE) (eight studies) where utilities, relative importance (RI) or ORs where used as units of measurement to quantify values and preferences. ${ }^{21} 23$ 25-28 3435 The next most frequent methodology was the time-trade-off (TTO) approach in four studies. ${ }^{24} 293133$ Utilities, health state disutilities and RI were the units of measurement in these studies. Other methodologies employed were willingness to pay, ${ }^{21}$ online surveys,${ }^{19}$ questionnaires,${ }^{30}$ crossover trials $^{2232}$ and case-note surveys ${ }^{20}$ (table 1$)$.

\section{Risk of bias and certainty of evidence assessment}

Overall, 12 studies were found at high-risk of bias due to the usage of non-validated instruments for eliciting preferences and invalid representation of efficacy and safety of the drug profiles. ${ }^{19-22}$ 24-28 303334 Only five studies were found at low risk of bias, these studies used a previously validated survey to measure preferences between different GLP-1 RA on both injection naive and experienced patients 2329313235 (figure 2).

We evaluated the certainty of evidence regarding the following drug profile comparisons: GLP-1 RA versus dypeptil peptidase-4 inhibitors (DPP-4i), insulin glargine and other glucose-lowering therapies, liraglutide versus exenatide and dulaglutide, dulaglutide versus semaglutide and studies evaluating attributes of GLP-1RA injection devices. The certainty of evidence was judged to be very low in all cases due to concerns regarding study design, risk of bias and imprecision in all cases. In addition, concerns regarding inconsistency and indirectness were identified in most of the evidence for the different drug profile comparisons (table 2).

\section{Preferences for GLP-1 RA versus other types of glucose- lowering medications}

Overall, five studies evaluated preferences for a GLP-1 RA versus other treatments of type 2 diabetes, such as insulin glargine,${ }^{23}$ sitagliptin, ${ }^{19}{ }^{20}$ vildagliptin, ${ }^{22}$ rosiglitazone and glimepiride. ${ }^{21}$ From these, one study was found to be at low risk of bias. ${ }^{23}$ Two studies were performed on the injectionnaïve population, ${ }^{1923}$ one on injection-experienced ${ }^{22}$ and 


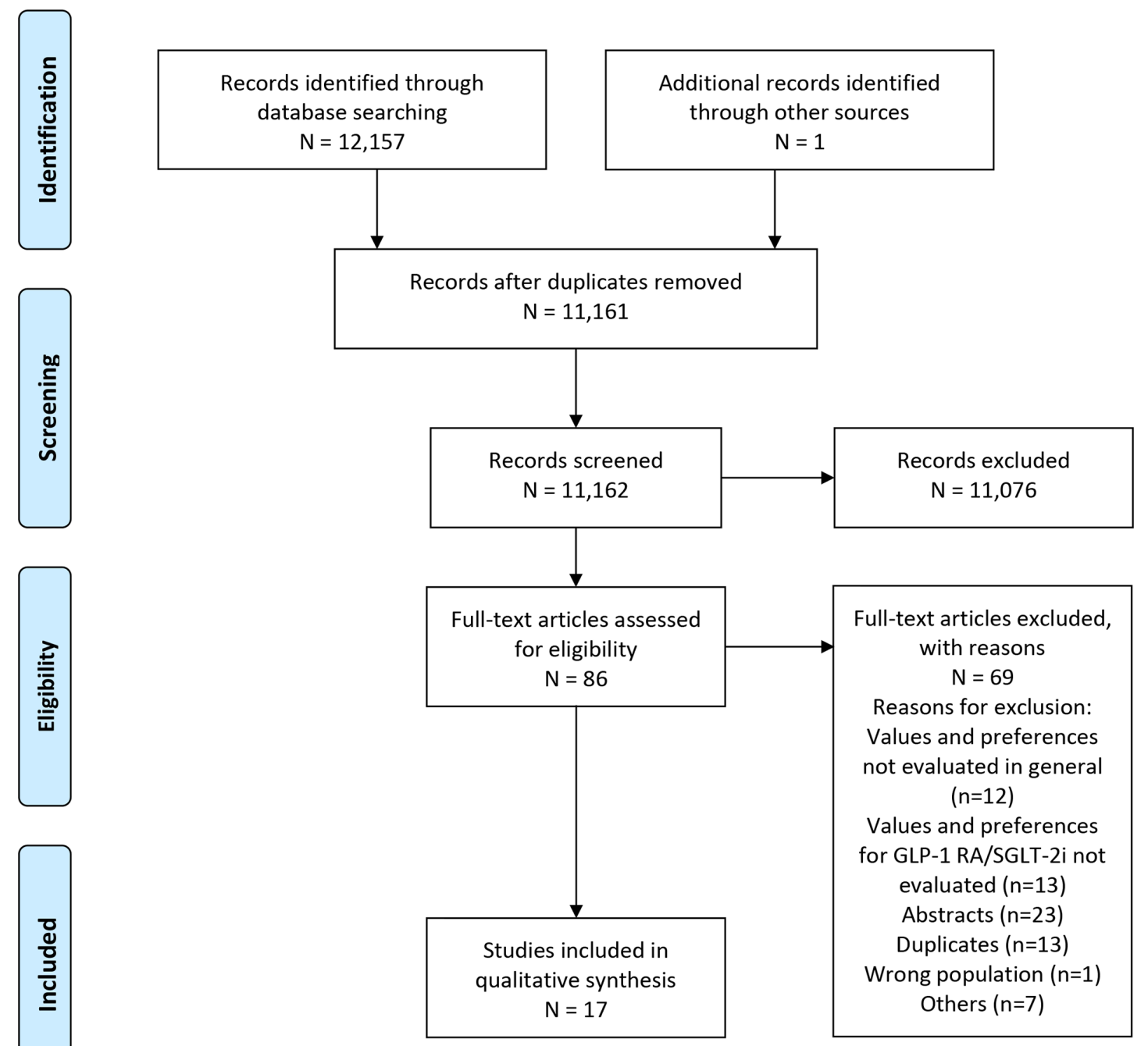

Figure 1 Study selection flow diagram. GLP-1 RA, glucagon-like peptide-1 receptor agonists; SGLT-2i, sodium-glucose cotransporter-2 inhibitors.

the remaining two on a mixed population. ${ }^{20}{ }^{21}$ Among the studies which presented drug profiles as part of their methodology, all studies described efficacy (defined as a change in glycosilated hemoglobin $[\mathrm{HbAlc}]$ ), proportion of side effects, weight change, dosing frequency and delivery system. Four studies described hypoglycaemic risk, ${ }^{19-21} 23$ and three included blood pressure change in the studied drugs profile. ${ }^{19-21}$ From the five studies, two described the all above-mentioned attributes on their drug profiles ${ }^{2021}$ (table 3 ). Shown below is a subdivision of the drug comparisons that were assessed in these studies.

\section{Glp-1 RA compared with DPP-4i}

Three studies evaluated preferences between orally administered DPP-4i (sitagliptin and vildagliptin) and GLP-1 RA (liraglutide). ${ }^{19} 2022$ Preference for DPP-4i in both injection naive and experienced patients was observed in two out of three studies. ${ }^{1922}$ Attributes ranked as the most important for choosing a DPP-4i over GLP-1 RA were its oral administration route and lesser frequency of side effects. For patients choosing GLP-1 RA, the most important attributes were blood sugar/HbAlc lowering effect and weight loss effect (table 4).

Insulin glargine compared with GLP-1 RA

Two studies evaluated preferences between liraglutide or dulaglutide and insulin glargine, both of them showed preference for GLP-1 RA. ${ }^{21}{ }^{23}$ The first study found that $75 \%$ of participants preferred a dulaglutide profile when compared with insulin glargine where among patients who preferred the former, the most important reasons were type of delivery system and dosing frequency, with RI (proportion of the variance in the medication decision accounted by each attribute) of $24.5 \%$ and $19.2 \%$ for each attribute, respectively. Moreover, in patients who preferred insulin, most important reasons for choice were lesser frequency of gastrointestinal adverse effects (RI: $45.3 \%$ ) and pancreatitis (RI: $26.5 \%){ }^{23}$ (table 4 )

In the second study (willingness-to pay-analysis), participants were prepared to pay an extra $€ 3.36 /$ day for liraglutide over insulin glargine where weight change was the most important attribute leading to liraglutide preference 


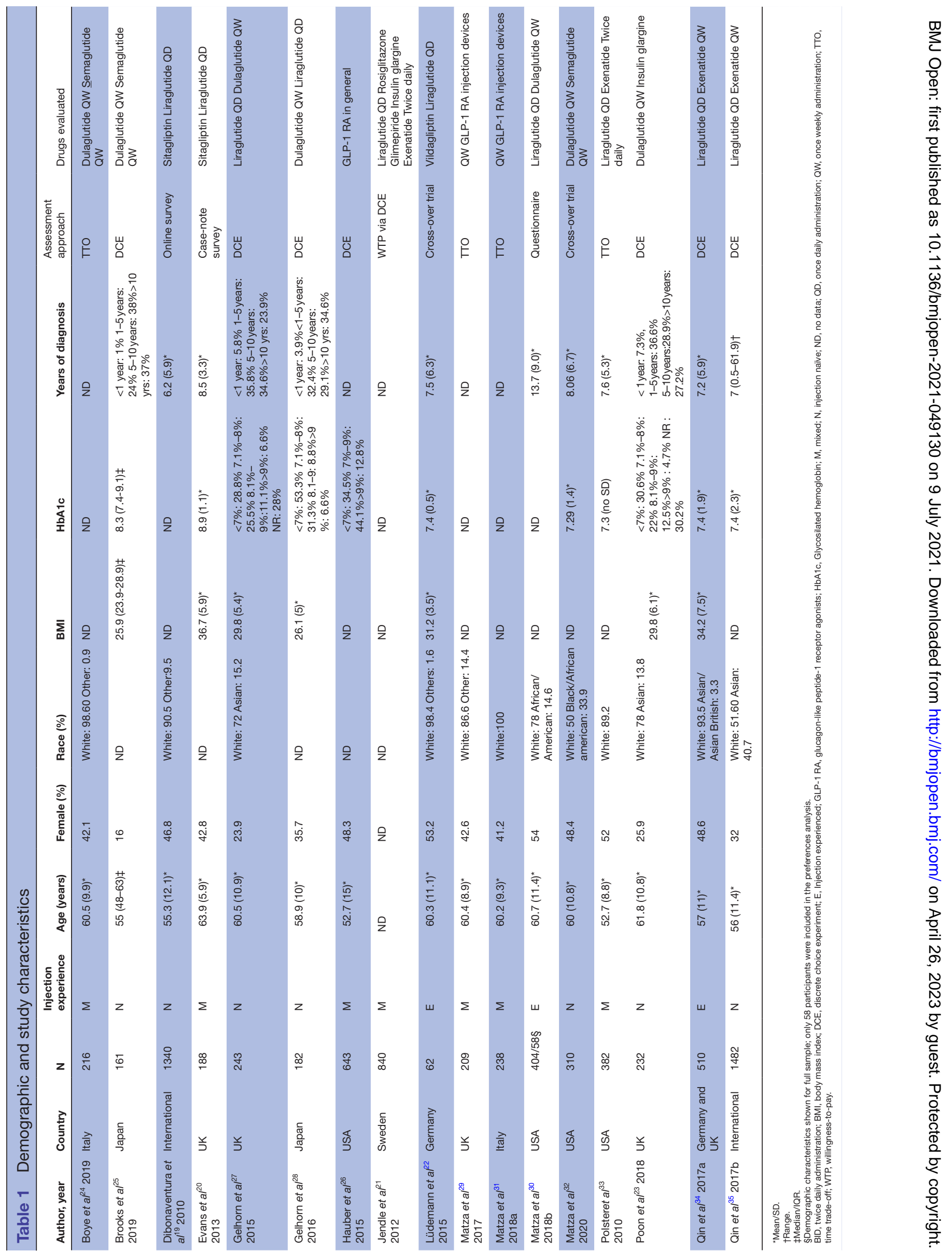




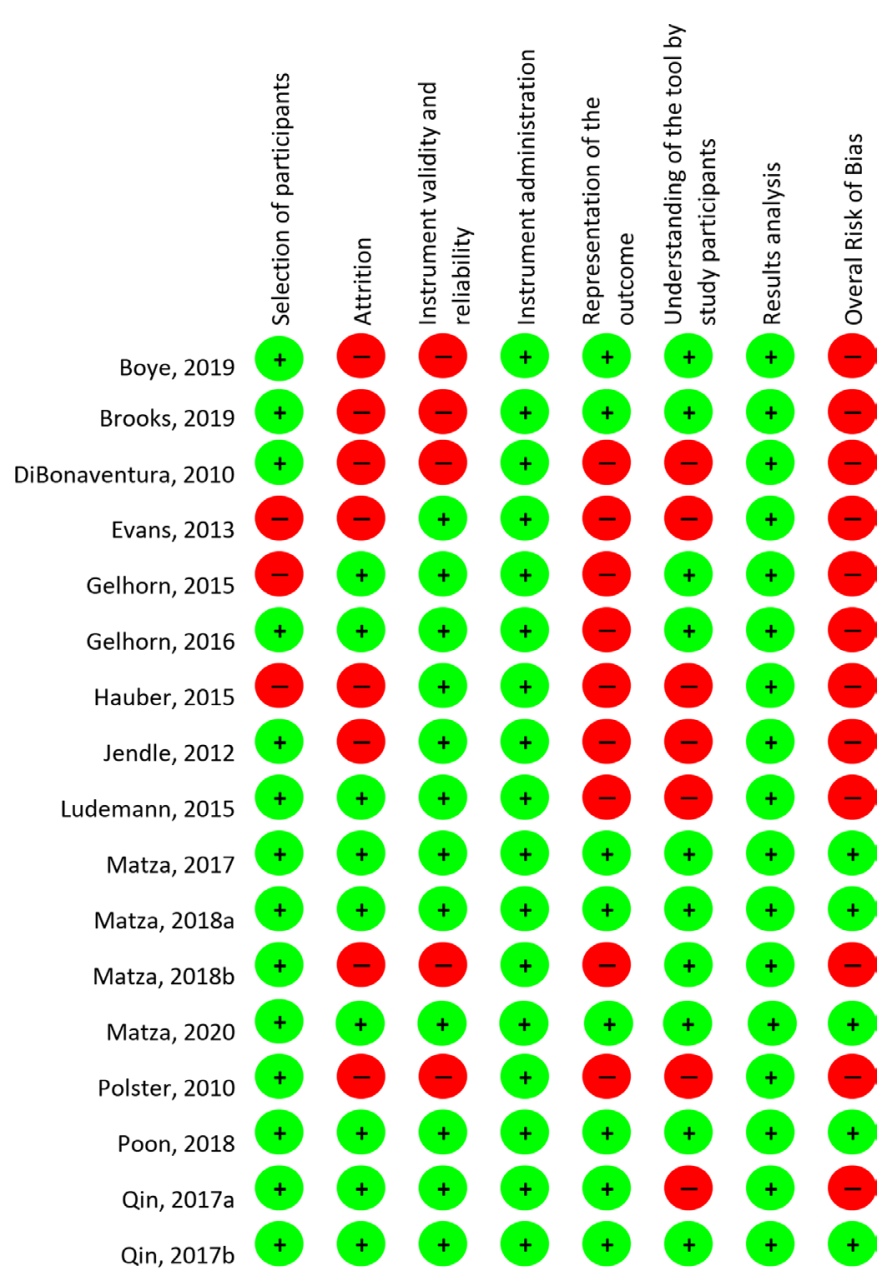

Low risk

High risk

Figure 2 Risk of bias assessment.

(€2.35/day). In this study, liraglutide was presented as the best profile among all subdomains. ${ }^{21}$ The risk for hypoglycaemic was not an important attribute for patients' preference in both studies.

\section{Other glucose-lowering treatments compared with GLP1-RA}

One study evaluated the preference for liraglutide and other oral treatments, including rosiglitazone and glimepiride. Participants were prepared to pay an extra €2.64 and $€ 1.94 /$ day for liraglutide over rosiglitazone and glimepiride, respectively. The main component for preference of liraglutide over both drugs was its weight loss effect. The only attribute which leads participants to pay more for rosiglitazone and glimepiride over liraglutide was the oral administration route. ${ }^{21}$

\section{Different GLP-1 RA medications}

Twelve studies evaluating preferences between different GLP-1 RA medications were included. Attributes that were included in these were related to dosing frequency and device type, but some also included efficacy, safety, and price as attributes. Drug profiles examined in these studies were extended release (weekly) and twice daily exenatide, once daily liraglutide and once weekly semaglutide and dulaglutide. Six of them were DCEs ${ }^{25-28} 3435$ and four were TTOs. ${ }^{24} 293133$ The remaining two were a questionnaire $^{30}$ and a cross-over trial. ${ }^{32}$

\section{Liraglutide versus exenatide}

Four studies evaluated this comparison. ${ }^{21} 33-35$ Overall, participants preferred once daily liraglutide compared with twice daily exenatide. However, they preferred once weekly exenatide compared with once daily liraglutide.

One survey found that $96 \%$ of included participants preferred once daily liraglutide over twice daily exenatide, where liraglutide also was presented as the drug having better efficacy, less rates of nausea and hypoglycaemic. ${ }^{33}$ Two other surveys (one on injection naive and the other on injection experienced users) reported that when assuming equal efficacy within both profiles (1.2 decreases in $\mathrm{HbA} 1 \mathrm{c}), 78.6 \%$ of injection experienced users preferred once weekly exenatide compared with a profile matching liraglutide. ${ }^{34}$ Among injection-naive participants, $77 \%$ preferred the profile matching exenatide. ${ }^{35}$ In both studies, attributes determining preference were better efficacy, lesser frequency of side effects and weekly dosing frequency. Moreover, even when efficacy was assumed to be better for liraglutide (1.2 vs 0.8 decrease in $\mathrm{HbA1c}$ ), patients still preferred a weekly exenatide matching profile. (table 4) A willingness-to-pay analysis demonstrated that participants were willing to pay an extra $€ 0.81 /$ day for once daily liraglutide over twice daily exenatide where once daily administration (lesser dosing frequency) was the main component driving the preference $(€ 1.04 /$ day $){ }^{21}$

\section{Liraglutide versus dulaglutide}

Three studies evaluated this comparison, one of them only compared device characteristics. ${ }^{272830}$ A preference for dulaglutide was observed in all three.

In two studies, one in Japan and the other in the UK most of the population preferred the profile representing dulaglutide $(94.5 \%$ and $83.1 \%$ for Japanese and UK population, respectively). Its profile consisted of a once weekly injection with a single-use prefilled pen compared with a once daily application with a multiuse pen that required dose titration for liraglutide. Slightly greater efficacy (reported difference in proportions of patients reaching treatment goals across groups was $<3 \%$ ), greater weight loss effect, and lesser frequency of nausea and hypoglycaemic were also attributes included on the dulaglutide profile. In both samples, the most important attributes for choosing a medication were dosing frequency (RI: $41.6 \%, 44.1 \%$ for the UK and Japanese population, respectively) and type of delivery system (RI: $35.5 \%, 26.3 \%$ for the UK and Japanese population, respectively) ${ }^{27} 28$ (table 4 ). In the third one, a survey comparing medication devices was applied on patients experienced to both treatments and revealed a 


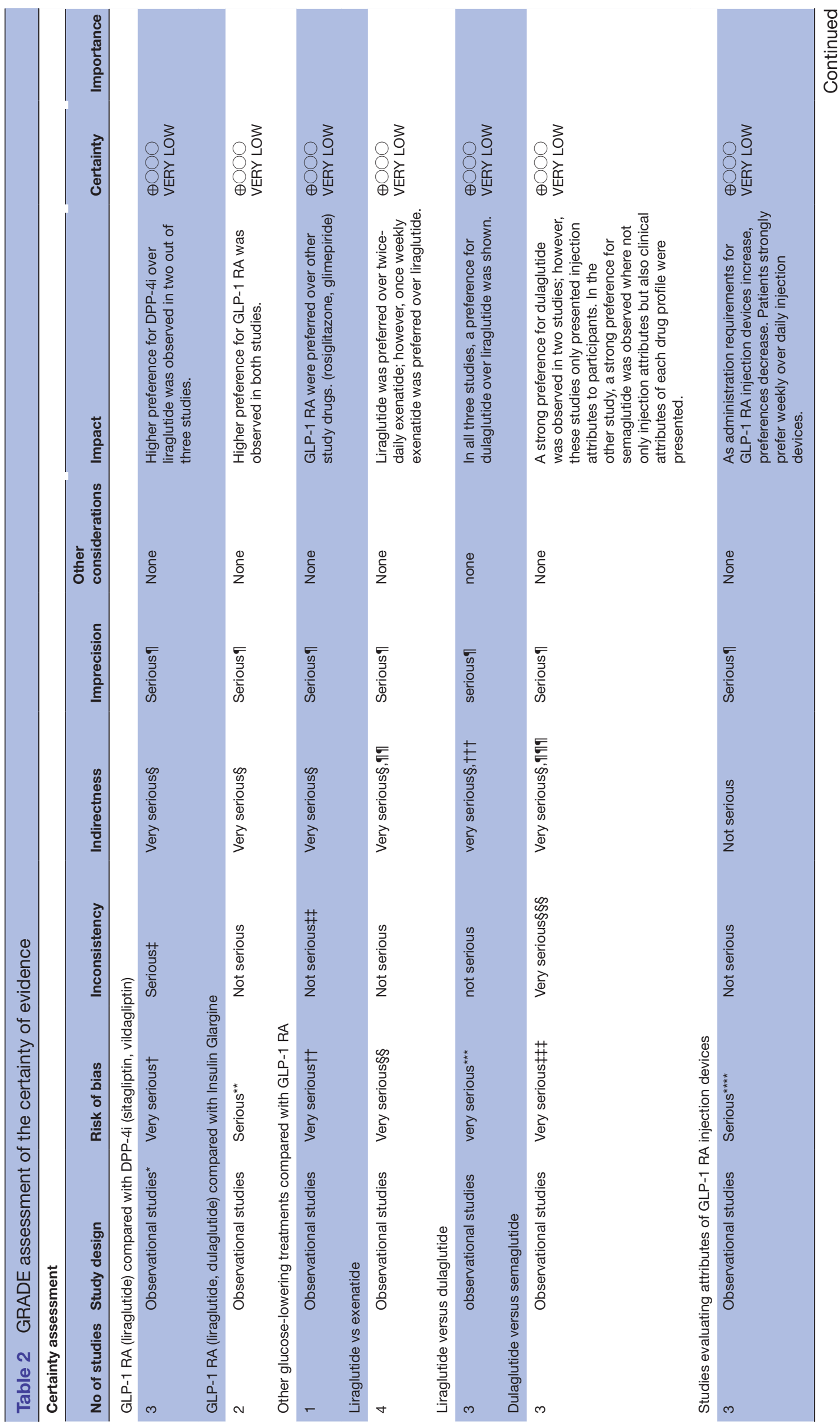

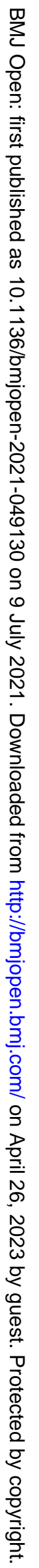


preference for the dulaglutide device (table 4). In this case, participants' preference was chosen based on their own experience. ${ }^{30}$

\section{Dulaglutide versus semaglutide}

Three studies evaluated this comparison where two of them evaluated device attributes ${ }^{24} 32$ and the other added clinical attributes to the drug profiles. ${ }^{25}$ Overall, among devices, participants preferred the one accompanying dulaglutide. When clinical attributes when considered in the drug profile, participants preferred semaglutide.

In a survey comparing device characteristics by providing hypothetical health states with each one, $88 \%$ of participants preferred the health state with the dulaglutide device over the semaglutide device, as the first one was considered 'less complicated' and 'quicker'. Considering that the study exclusively analysed preferences regarding injection devices, no information regarding efficacy, side effects and price was assessed on either of the health states, assuming that they were all equal regarding these characteristics. Dulaglutide consisted of a one-dose injection with no needle handling and no dose adjustment. Patients who preferred semaglutide profile considered that a one-dose injection would make them 'buy too many pens'. ${ }^{24}$ A cross-over trial comparing both injection devices found that $84.2 \%$ of participants preferred the dulaglutide profile, mainly due to its 'ease of use'. ${ }^{32}$

In contrast, one study comparing both drugs using five attributes (method of administration, HbA1c change, reduction in cardiovascular (CV) risk, weight change and common side effects) reported that $80 \%$ of participants preferred the semaglutide profile, which was presented as the more efficient ( $1.9 \%$ vs $1.4 \%$ reduction in $\mathrm{HbAlc}$ ), with greater weight loss effect, greater rate of nausea, $26 \%$ $\mathrm{CV}$ risk reduction (vs no risk reduction for dulaglutide), and with a multidose prefilled pen with dose adjustment (vs a single-dose prefilled pen with no dose adjustment representing dulaglutide). $\mathrm{CV}$ risk reduction followed by HBA1c reduction and rate of side effects were the most important attributes leading to their choice based on coefficient utilities $^{25}$ (table 4 ).

\section{Studies evaluating attributes of GLP-1 RA injection devices and administration regimes}

Three studies fell into this category, none of which evaluated a specific drug profile; conversely, these studies evaluated patients' preferences for injection devices based on different device attributes (table 4). One found that among a mixed population of injection naïve and injection experienced patients, changing injection frequency from daily to weekly was the most important attribute for choice of treatment. ${ }^{26}$ The other two found consistent main findings; each administration requirement (needle handling, reconstitution and waiting) was associated with higher disutilities when compared with an oral health state. $^{29} 31$ 


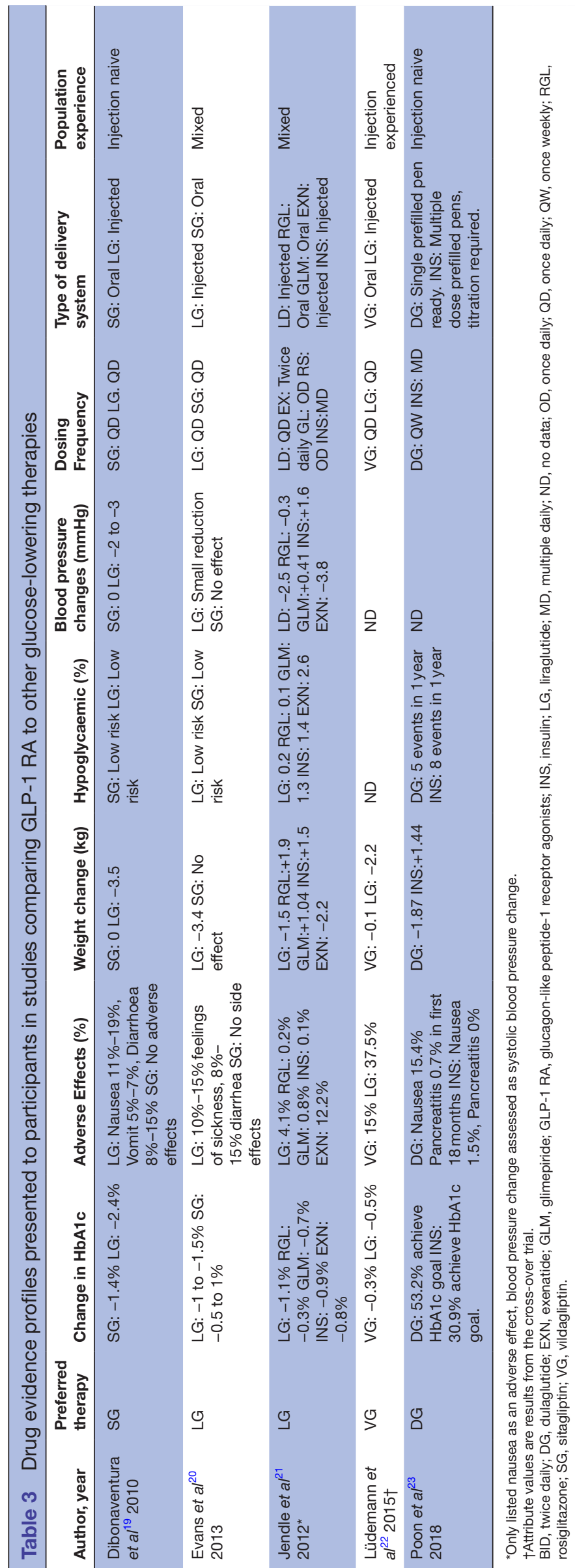




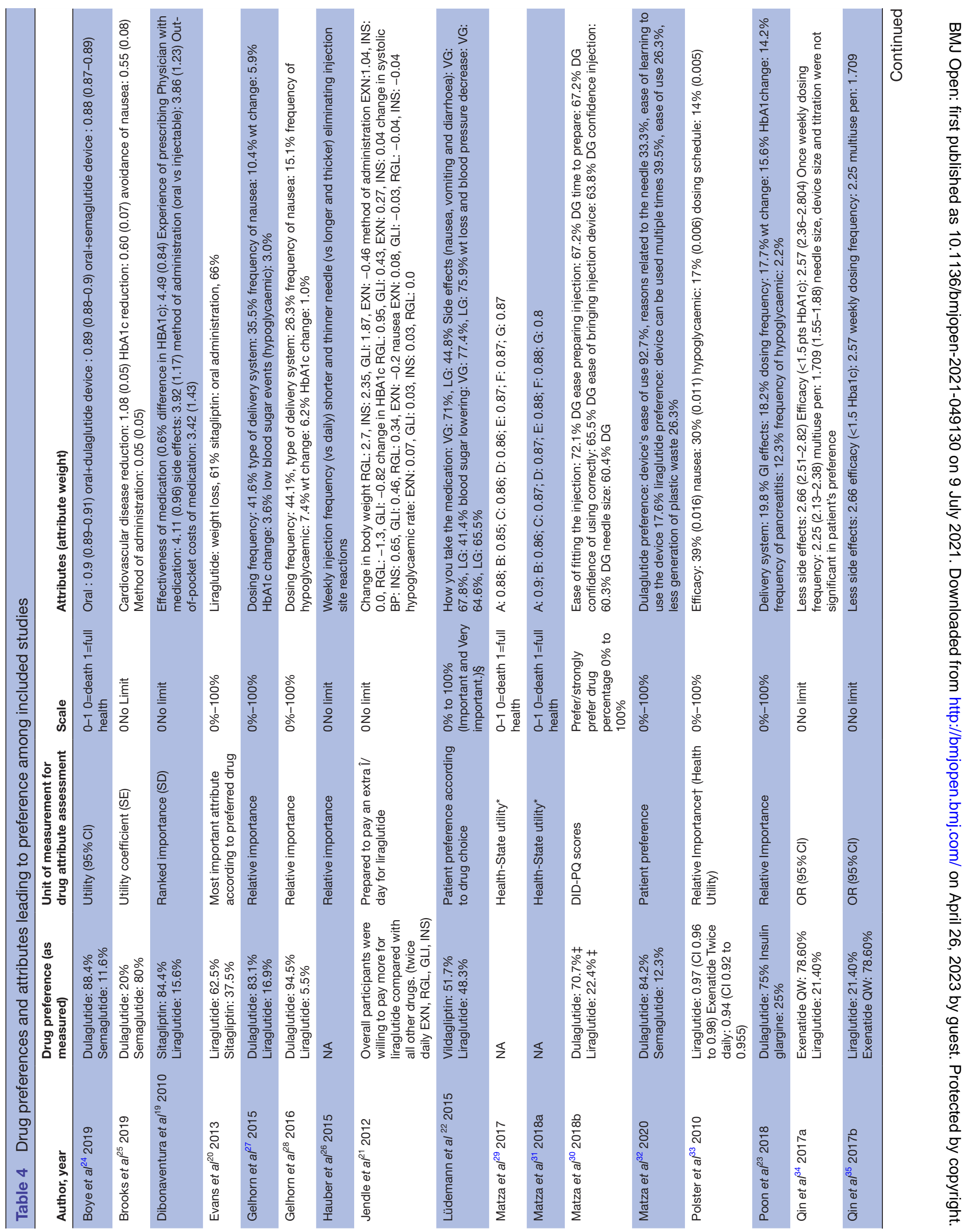


In this systematic review, we found no direct evidence to inform judgements about how patients with type 2 diabetes considering SGLT-2i and GLP-1 RA value established benefits on cardiovascular and kidney outcomes, weighed against harms and burdens of treatments. Taking this into account, several evidence evaluating preferences for GLP-1 RA was found where patients consistently showed resistance to injectables and complicated devices, preferring oral medications or weekly injected devices, which reflects on potential burdens of treatment likely to impact their treatment choices. However, these results demonstrate a major shortcoming of our systematic review; none of the studies presented patients with best current evidence on benefits and harms of these drugs, making any inferences about values and preferences of highly limited value as analysing the state of evidence on a certain medication at a specific point in time does not necessarily reflect the state of the same in the future with respect to it, therefore, treatment profiles could vary depending on the year in which the preference study was performed. Furthermore, studies defined efficacy of different drugs based on their glucose-lowering potential and for almost all did not assess patient-important microvascular or macrovascular outcomes. ${ }^{36}$

The evidence on burden of treatment serves as a reminder to guideline panels often restricting judgements of values and preferences to benefits and harms and clinicians leaving this factor out of the equation in assisting patients in making well-informed treatment choices. $^{2}$ Indeed, the BMJ Rapid Recommendations put great emphasis on this evidence, directly impacting recommendations favouring SGLT-2i over GLP-1 RA.

This review has multiple strengths. We used of a previously validated search strategy to perform systematic reviews and meta-analysis of patients' preferences studies. Additionally, we followed high methodological standards in conducting the review and evaluated each study's quality with a specialised tool for patients' preference studies and performed a further comprehensive analysis of the certainty of evidence by following the GRADE working group constructs. Finally, we considered the consistency of the evidence presented in the included studies to elicit patients' preferences with the current best available evidence when drawing conclusions. This approach emphasised issues about the applicability of findings of this review to the BMJ Rapid Recommendations (Box 1).

We acknowledge there are several important limitations in our study. Our results are based mostly on studies graded at high risk of bias due to important methodological concerns. As a result, when assessing the certainty of evidence, all preferences in each drug comparison are graded at a very low certainty. More importantly, most of the included studies drew conclusions that could be influenced by conflict of interest. Moreover, there was no information regarding other important second-line treatments for diabetes such as SGLT-2i, therefore, we could 
not directly establish preferences between SGLT-2i and GLP-1 RA which would be very important due to both drugs' increasing popularity among patients and clinicians. Some explanations on the absence of studies evaluating preferences for and among SGLT-2i could be that they are relatively new when compared with GLP-1 RA (the first SGLT-2i to be approved by the Food and Drug Administration was canagliflozin in 2013, compared with exenatide in 2005) and that as GLP-1 RA tend to have similar efficacy profiles, industry-based studies could have been carried out to assess preferences between treatments based on other attributes.

Overall, there is still not enough evidence to demonstrate a patient preference tendency between GLP-1 RA and SGLT-2i. Clinicians should individualise the use of these medications to each patient individual context, taking into consideration the best current evidence on efficacy and side effects all the while considering treatment burden, patient preferences, among other factors in the process of shared decision making. Furthermore, when opting to use GLP-1 RA, it would be optimal to consider weekly versions due to higher preferences observed for these in the present study.

Further studies are needed to elicit patients' values and preferences among wider spectrum of oral and injectable diabetes treatments. There is a specific and urgent need to assess patient's values and preferences between weekly injected GLP-1 RAs and all other classes of oral glucoselowering medications including SGLT-2i. Furthermore, our review highlights the need for information about treatment efficacy based on systematic reviews rather than single studies. Additionally, our review findings emphasise the importance of standardising the way in which drug profiles are presented in values and preferences studies, where we suggest that attributes such as efficacy, side effects, mode of administration and dosage, cost, among other important variables to be constantly included in the building of drug profiles so that precise and trustworthy results are ensured.

\section{Author affiliations}

${ }^{1}$ Plataforma INVEST Medicina UANL-KER Unit Mayo Clinic (KER Unit Mexico), Universidad Autonoma de Nuevo Leon Facultad de Medicina, Monterrey, Nuevo León, Mexico

${ }^{2}$ Endocrinology Division, Department of Internal Medicine, Hospital Universitario Dr José Eleuterio González, Monterrey, Nuevo León, Mexico

${ }^{3}$ Department of Health Research Methods, Evidence, and Impact, McMaster University, Hamilton, Ontario, Canada

${ }^{4}$ Internal Medicine, Division of Nephrology and Hypertension, University of Kansas

Medical Center, Kansas City, Kansas, USA

${ }^{5}$ Department of Medicine, University of Otago, Christchurch, Christchurch, New Zealand

${ }^{6}$ Department of Endocrinology and Metabolism, West China Hospital, Sichuan University, Chengdu, Sichuan, China

${ }^{7}$ Division of Population Health and Genomics, Ninewells Hospital and School of Medicine, University of Dundee, Dundee, UK

${ }^{8}$ The center of Gerontology and Geriatrics, National Center for Geriatric Clinical Research, Sichuan University, Chengdu, Sichuan, China

${ }^{9}$ Department of Medicine, Lovisenberg Diakonale Hospital, Oslo, Norway
Contributors Conceiving of the research idea: JGG-G, RR-G, LL, RAM, SP, SL, QH and PV; First draft of the research protocol: JGG-G, ADG-C, JMM-A, RCS, NA-V, RRG; Final version of the research protocol: all authors; Search strategy design: NA-V; Study selection process: JGG-G, ADG-C, JMM-A and RCS; Data extraction process: JGG-G, ADG-C, JMM-A and RCS; Data synthesis: JGG-G, ADG-C, JMM-A and RCS; First draft of the manuscript: JGG-G, ADG-C, JMM-A, RCS and RR-G; Final version of the manuscript: all authors.

Funding The authors have not declared a specific grant for this research from any funding agency in the public, commercial or not-for-profit sectors.

Competing interests All authors have completed the ICMJE uniform disclosure form at www.icmje.org/coi_disclosure.pdf and declare: JGG-G, ADG-C, JMM-A, LL, RCS, RAM, SP, SL, QH, NA-V, PV, RR-G: no support from any organisation for the submitted work; no financial relationships with any organisations that might have an interest in the submitted work in the previous three years. SL was supported by grants from the National Natural Science Foundation of China (grant number 21534008), Sichuan Science and Technology Program (grant number 2019YFH0150), and 1.3.5 Project for Disciplines of Excellence, West China Hospital, Sichuan University (grant number ZYGD18022 and 2020HXF011). But none of the grant contributes to this work.

Patient and public involvement statement The paper informs a Rapid Recommendation on the use of SGLT-2 inhibitors and GLP-1 receptor agonists that will be released on a digital platform (www.magicproject.org) and made available to organisations to adapt for their own materials and purposes.

Patient consent for publication Not required.

Provenance and peer review Not commissioned; externally peer reviewed.

Data availability statement № additional data available.

Supplemental material This content has been supplied by the author(s). It has not been vetted by BMJ Publishing Group Limited (BMJ) and may not have been peer-reviewed. Any opinions or recommendations discussed are solely those of the author(s) and are not endorsed by BMJ. BMJ disclaims all liability and responsibility arising from any reliance placed on the content. Where the content includes any translated material, BMJ does not warrant the accuracy and reliability of the translations (including but not limited to local regulations, clinical guidelines, terminology, drug names and drug dosages), and is not responsible for any error and/or omissions arising from translation and adaptation or otherwise.

Open access This is an open access article distributed in accordance with the Creative Commons Attribution Non Commercial (CC BY-NC 4.0) license, which permits others to distribute, remix, adapt, build upon this work non-commercially, and license their derivative works on different terms, provided the original work is properly cited, appropriate credit is given, any changes made indicated, and the use is non-commercial. See: http://creativecommons.org/licenses/by-nc/4.0/.

\section{ORCID iDs}

Juan Manuel Millán-Alanís http://orcid.org/0000-0001-5583-2582

Ricardo Cesar Solis http://orcid.org/0000-0001-7206-6955

Qiukui Hao http://orcid.org/0000-0002-5160-5144

René Rodríguez-Gutiérrez http://orcid.org/0000-0002-3022-5302

\section{REFERENCES}

1 Inzucchi SE, Bergenstal RM, Buse JB, et al. Management of hyperglycemia in type 2 diabetes: a patient-centered approach: position statement of the American diabetes association (ADA) and the European association for the study of diabetes (EASD). Diabetes Spectrum 2012;25:154-71.

2 Stiggelbout AM, Van der Weijden T, De Wit MPT, et al. Shared decision making: really putting patients at the centre of healthcare. BMJ 2012;344:e256.

3 Marso SP, Bain SC, Consoli A, et al. Semaglutide and cardiovascular outcomes in patients with type 2 diabetes. $N$ Engl J Med 2016;375:1834-44.

4 Marso SP, Daniels GH, Brown-Frandsen K, et al. Liraglutide and cardiovascular outcomes in type 2 diabetes. New England Journal of Medicine 2016;375:311-22.

5 Bethel MA, Patel RA, Merrill P, et al. Cardiovascular outcomes with glucagon-like peptide-1 receptor agonists in patients with type 2 diabetes: a meta-analysis. Lancet Diabetes Endocrinol 2018;6:105-13.

6 Ahrén B, Masmiquel L, Kumar H, et al. Efficacy and safety of onceweekly semaglutide versus once-daily sitagliptin as an add-on 
to metformin, thiazolidinediones, or both, in patients with type 2 diabetes (sustain 2): a 56-week, double-blind, phase 3A, randomised trial. Lancet Diabetes Endocrinol 2017;5:341-54.

7 Diamant M, Van Gaal L, Stranks S, et al. Once Weekly exenatide compared with insulin glargine titrated to target in patients with type 2 diabetes (DURATION-3): an open-label randomised trial. Lancet 2010;375:2234-43.

8 Fonseca VA, Alvarado-Ruiz R, Raccah D, et al. Efficacy and safety of the once-daily GLP-1 receptor agonist lixisenatide in monotherapy: a randomized, double-blind, placebo-controlled trial in patients with type 2 diabetes (GetGoal-Mono). Diabetes Care 2012;35:1225-31.

9 Hernandez AF, Green JB, Janmohamed S, et al. Albiglutide and cardiovascular outcomes in patients with type 2 diabetes and cardiovascular disease (harmony outcomes): a double-blind, randomised placebo-controlled trial. Lancet 2018;392:1519-29.

10 Marre M, Shaw J, Brändle M, et al. Liraglutide, a once-daily human GLP-1 analogue, added to a sulphonylurea over 26 weeks produces greater improvements in glycaemic and weight control compared with adding rosiglitazone or placebo in subjects with type 2 diabetes (LEAD-1 SU). Diabet Med 2009;26:268-78.

11 Thieu VT, Robinson S, Kennedy-Martin T, et al. Patient preferences for glucagon-like peptide 1 receptor-agonist treatment attributes. Patient Prefer Adherence 2019;13:561-76.

12 Siemieniuk RA, Agoritsas T, Macdonald H, et al. Introduction to BMJ rapid recommendations. BMJ 2016;354:i5191.

13 Shamseer L, Moher D, Clarke M, et al. Preferred reporting items for systematic review and meta-analysis protocols (PRISMA-P) 2015: elaboration and explanation. BMJ 2015;349:g7647.

14 Selva A, Sanabria AJ, Pequeño S, et al. Incorporating patients' views in Guideline development: a systematic review of guidance documents. J Clin Epidemiol 2017;88:102-12.

15 Andrews J, Guyatt G, Oxman AD, et al. Grade guidelines: 14 . going from evidence to recommendations: the significance and presentation of recommendations. J Clin Epidemiol 2013;66:719-25.

16 Joy SM, Little E, Maruthur NM, et al. Patient preferences for the treatment of type 2 diabetes: a scoping review. Pharmacoeconomics 2013;31:877-92.

17 Zhang Y, Alonso-Coello P, Guyatt GH, et al. Grade guidelines: 19. assessing the certainty of evidence in the importance of outcomes or values and preferences-Risk of bias and indirectness. J Clin Epidemiol 2019;111:94-104.

18 Murad MH, Mustafa RA, Schünemann $\mathrm{HJ}$, et al. Rating the certainty in evidence in the absence of a single estimate of effect. Evid Based Med 2017;22:85-7.

19 Dibonaventura MD, Wagner J-S, Girman CJ, et al. Multinational Internet-based survey of patient preference for newer oral or injectable type 2 diabetes medication. Patient Prefer Adherence 2010;4:397.

20 Evans M, McEwan P, O'Shea R, O'Shea R, et al. A retrospective, case-note survey of type 2 diabetes patients prescribed incretinbased therapies in clinical practice. Diabetes Ther 2013;4:27-40.

21 Jendle J, Torffvit O, Ridderstråle M. Willingness to pay for diabetes drug therapy in type 2 diabetes patients: based on lead clinical programme results. J Med Econ 2012;15:1-5.

22 Lüdemann J, Dütting ED, Dworak M. Patient preference and tolerability of a DPP-4 inhibitor versus a GLP-1 analog in patients with type 2 diabetes mellitus inadequately controlled with metformin: a 24-week, randomized, multicenter, crossover study. Ther Adv Endocrinol Metab 2015;6:141-8.
23 Poon JL, Boye KS, Thieu VT. Preferences for attributes of medications among patients with type 2 diabetes: a crossmedication class comparison of injection therapies. Curr Res Diabetes Obes J 2018;6:1-13.

24 Boye KS, Matza LS, Stewart KD, et al. Patient preferences and health state utilities associated with dulaglutide and semaglutide injection devices among patients with type 2 diabetes in Italy. J Med Econ 2019;22:806-13.

25 Brooks A, Langer J, Tervonen T, et al. Patient preferences for GLP-1 receptor agonist treatment of type 2 diabetes mellitus in Japan: a discrete choice experiment. Diabetes Ther 2019;10:735-49.

26 Hauber AB, Nguyen $\mathrm{H}$, Posner J, et al. A discrete-choice experiment to quantify patient preferences for frequency of glucagon-like peptide-1 receptor agonist injections in the treatment of type 2 diabetes. Curr Med Res Opin 2016;32:251-62.

27 Gelhorn HL, Poon J-L, Davies EW, et al. Evaluating preferences for profiles of GLP-1 receptor agonists among injection-naïve type 2 diabetes patients in the UK. Patient Prefer Adherence 2015;9

28 Gelhorn HL, Bacci ED, Poon JL, et al. Evaluating preferences for profiles of glucagon-like peptide-1 receptor agonists among injection-naive type 2 diabetes patients in Japan. Patient Prefer Adherence 2016;10:1337-48.

29 Matza LS, Boye KS, Stewart KD, et al. Health state utilities associated with attributes of Weekly injection devices for treatment of type 2 diabetes. BMC Health Serv Res 2017;17:1-10.

30 Matza LS, Boye KS, Currie BM, et al. Patient perceptions of injection devices used with dulaglutide and liraglutide for treatment of type 2 diabetes. Curr Med Res Opin 2018;34:1457-64.

31 Matza LS, Boye KS, Jordan JB, et al. Patient preferences in Italy: health state utilities associated with attributes of Weekly injection devices for treatment of type 2 diabetes. Patient Prefer Adherence 2018;12:971-9.

32 Matza LS, Boye KS, Stewart KD, et al. Assessing patient preference between the dulaglutide Pen and the semaglutide Pen: a crossover study (prefer). Diabetes Obes Metab 2020;22:355-64.

33 Polster M, Zanutto E, McDonald S, et al. A comparison of preferences for two GLP-1 products--liraglutide and exenatide--for the treatment of type 2 diabetes. J Med Econ 2010;13:655-61.

34 Qin L, Chen S, Flood E, et al. Glucagon-Like peptide-1 receptor agonist treatment attributes important to injection-experienced patients with type 2 diabetes mellitus: a preference study in Germany and the United Kingdom. Diabetes Ther 2017;8:335-53.

35 Qin L, Chen S, Flood E, et al. Glucagon-Like peptide-1 receptor agonist treatment attributes important to injection-naïve patients with type 2 diabetes mellitus: a multinational preference study. Diabetes Ther 2017;8:321-34.

36 Rodríguez-Gutiérrez R, Montori VM. Glycemic control for patients with type 2 diabetes mellitus: our evolving faith in the face of evidence. Circ Cardiovasc Qual Outcomes 2016;9:504-12.

37 Li S, Vandvik PO, Hao Q. Sodium-Glucose cotransporter-2 inhibitors (SGLT-2i) glucagon-like peptide-1 (GLP-1) receptor agonists for adults with type 2 diabetes at different risk of cardiovascular and renal outcomes: a clinical practice guideline. BMJ.

38 Buchan T, Malik A, Chan C, et al. Risk prediction models for cardiovascular and renal outcomes in patients with type 2 diabetes: a systematic review. BMJ.

39 Palmer SC, Tendal B, Mustafa RA, et al. Sodium-Glucose transport protein 2 inhibitors and GLP-1 receptor agonists for type 2 diabetes: a systematic review and network meta-analysis of randomised controlled trials. BMJ. 